\title{
CHEMICAL EVOLUTION OF \\ STAR-FORMING VISCOUS DISKS
}

\author{
Y. SHIOYA AND M. CHIBA \\ Astronomical Institute, Tohoku University \\ Aoba-ku, Sendai 980-77, JAPAN
}

Star-forming viscous disk model was proposed by Lin \& Pringle (1987) as an ubiquitous mechanism to form the exponential stellar disk of spiral galaxies. To clarify whether the viscous evolution models can explain the chemical properties of general spiral galaxies, we have studied the chemical evolution of star-forming viscous disks under the several physical conditions in relation to the possible processes of the disk formation, and compared with the observed gas and metallicity distributions of spiral galaxy disks.

First, we compared the abundance gradient predicted our models with those observed for spiral galaxies (Zaritsky et al. 1994). In most cases, the model abundance gradient is consistent with the observed gradient. Second, we studied the relation between the gas mass fraction and metallicity $\left(f_{g}\right.$ - $Z$ relation). We found that there are two distinct classes in the chemical properties of spiral galaxy disks: for the galaxies classified as the class A, their $f_{g}-Z$ relations are similar to the curve given by the Simple model with fixed $y$ as well as by viscous disk models in all radii, whereas for the class B their gas mass fractions are larger than the model prediction for the corresponding metallicity at the molecular gas dominant region. This fact implies that the time scale of star formation is larger than the viscous time scale in molecular gas dominant regions. If so, it is suggested that the value of the viscosity changes with physical condition of gaseous phase: diffuse gas or dense clouds.

We conclude that the chemical evolution of the star-forming viscous disk, which holds the physical basis from the theory of disk formation, is generally consistent with the chemical properties of the Milky Way and external disk galaxies - except the apparent discrepancy for moleculargas dominated galaxies. For further understanding, it is thus necessary to consider the role of molecule formation and/or destruction in evolution of spiral galaxies. 